\title{
miR-130b-5p promotes proliferation, migration and invasion of gastric cancer cells via targeting RASAL1
}

\author{
HONG CHEN, YIQIONG YANG, JING WANG, DUO SHEN, JIYI ZHAO and QIAN YU \\ Department of Gastroenterology, Zhongda Hospital, Medical School, \\ Southeast University, Nanjing, Jiangsu 210000, P.R. China
}

Received August 11, 2016; Accepted December 22, 2017

DOI: $10.3892 / \mathrm{ol} .2018 .8174$

\begin{abstract}
The aim of the present study was to investigate the targeted interaction between microRNA (miR)-130b-5p and RAS protein activator like 1 (RASAL1) gene and elucidate the function of miR-130b-5p in cell proliferation, migration and invasion in gastric cancer. Expression of miR-130b-5p and RASAL1 in seven gastric cell lines was detected by reverse transcription-quantitative polymerase chain reaction (RT-qPCR). MGC803 cells were selected for further study since they exhibited a marked increase in expression of miR-130b-5p accompanied by decreased expression of RASAL1. MGC803 cells were transfected with miR-130b-5p mimics and miR-130b-5p inhibitor using Lipofectamine 2000 for over- and underexpression, respectively, with cells transfected with negative control (NC) sequence as the control. In addition, a luciferase reporter gene assay was performed to evaluate the targeted interaction between miR-130b-5p and RASAL1. Then, alterations in RASAL1 expression were detected by RT-qPCR and western blot analysis following transfection with miR-130b-5p mimics and miR-130b-5p inhibitor. Cell proliferation, colony formation, and migration and invasion ability were detected by MTT, colony formation and Transwell assays, respectively. RASAL1 was demonstrated to be a target gene of miR-130b-5p by luciferase reporter gene assay. In addition, the expression of RASAL1 was significantly lower in MGC803 cells that were transfected with miR-130b-5p mimics and significantly higher in cells transfected with miR-130b-5p inhibitor in comparison with cells transfected with $\mathrm{NC}(\mathrm{P}<0.05)$. Furthermore, the experimental group transfected with miR-130b-5p mimics manifested significantly higher cell proliferation, increased colony formation and increased migratory and invasive capacities $(\mathrm{P}<0.05)$. By contrast, cells transfected with miR-130b-5p inhibitor exhibited
\end{abstract}

Correspondence to: Professor Hong Chen, Department of Gastroenterology, Zhongda Hospital, Medical School, Southeast University, 87 Dingjiaqiao, Gulou, Nanjing, Jiangsu 210000, P.R. China

E-mail: njchenhong66@163.com

Key words: microRNA-130b, RAS protein activator like 1, gastric cancer significantly lower cell proliferation, decreased colony formation and decreased migratory and invasive capacities, compared with cells transfected with $\mathrm{NC}(\mathrm{P}<0.05)$. In conclusion, RASAL1 was demonstrated to be a target gene of miR-130b-5p. Overexpression of miR-130b-5p results in promoted proliferation, colony formation and migration and invasion abilities through targeted modulation of RASAL1.

\section{Introduction}

Gastric cancer is one of the most common types of adenocarcinoma and the second leading cause of cancer-associated cases of mortality worldwide, despite having a low incidence in developed countries (1). The therapy for stomach cancer is an integrated process that involves surgery, radiation treatment and chemotherapy (2). It has been acknowledged that the effectiveness of treatment of tumors is associated with TNM-staging (3); however, patients with gastric carcinoma may be treated with radical resection, but the 5-year survival rates of patients are low due to the invasiveness and drug resistance of cancer cells (4). Thus, there is an urgent requirement to study the molecular mechanisms of gastric carcinoma (5). MicroRNAs (miRNAs) are small, non-coding RNAs composed of 18-25 nucleotides, which function through binding the 3'-untranslated region (3'-UTR) of targeted mRNAs, eventually leading to decreased gene expression and translation (6). It has been reported that abnormal levels of miRNAs are associated with the occurrence of cancer via affecting cell proliferation, apoptosis, metastasis and susceptibility to therapy (7). Therefore, studying the function of miRNAs may reveal novel treatment strategies for cancer.

miRNA (miR)-130b, situated in the 22q11 locus, has been demonstrated to have dual characteristics in the progression of tumors. For instance, it was identified to be an oncogene in gastric cancer, liver cancer, and endometrial cancer. By contrast, it was demonstrated to have a protective effect against ovarian and thyroid papillary carcinoma (8). These studies indicate that the role of miR-130b depends on the tumor classification. Our previous study identified that the expression of RAS protein activator like 1 (RASAL1) is decreased in gastric cancer in in vitro and in vivo experiments (9). However, the association between miR-130b-5p and RASAL1 and the effect of miR-130b-5p on gastric cancer has not previously been studied.

In the present study, an increase in miR-130b-5p and a decrease in RASAL1 were identified in gastric carcinoma cell 
lines. In addition, miR-130b-5p could facilitate the migration and invasion abilities of gastric cell lines. The role of miR-130b-5p in gastric cancer was associated with decreased RASAL1, which was demonstrated to be a novel target gene of miR-130b-5p. These findings could have implications for improving the treatment of gastric cancer.

\section{Materials and methods}

Cell lines and culture. The human gastric cell lines AGS, BGC823, MGC803, MKN45, MKN74, SGC7901 and GES-1 were purchased from Shanghai Institute of Digestive Disease (Shanghai, China) and were cultured in RPMI-1640 medium (Gibco; Thermo Fisher Scientific, Inc., Waltham, MA, USA), supplemented with $10 \%$ fetal bovine serum (Gibco; Thermo Fisher Scientific, Inc.), $100 \mu \mathrm{g} / \mathrm{ml}$ streptomycin and $100 \mathrm{U} / \mathrm{ml}$ penicillin (Gibco; Thermo Fisher Scientific, Inc.). Cells were cultured in a $37^{\circ} \mathrm{C}$ incubator with $5 \% \mathrm{CO}_{2}$.

Transfection of MGC803 cells with miR-130b-5p mimics and inhibitor. MGC803 cells were transfected with miR-130b-5p mimics (sense, 5'-ACUCUUUCCCUGUUGCACUAC-3' and antisense, 5'-AGUGCA ACAGGGAAAGAGUUU-3') and miR-130b-5p inhibitor (GUAGUGCAACAGGGAAAG AGU). Cells were transfected with NC (5'-UCACAACCU CCUAGAAAGAGU-3') as the control. miR-130b-5p mimics, miR-130b-5p inhibitor and NC were designed and synthesized by Shanghai GenePharma Co., Ltd. (Shanghai, China) and transfected into cells using Lipofectamine 2000 (Invitrogen; Thermo Fisher Scientific, Inc.), according to the manufacturer's protocol. Transfection was conducted for 1-2 days according to cell growth conditions prior to subsequent analysis. Each experiment was repeated at least three times.

Reverse transcription-quantitative polymerase chain reaction $(R T-q P C R)$. Expression of miR-130b-5p and RASAL1 were detected by RT-qPCR. TRIzol (Invitrogen; Thermo Fisher Scientific, Inc.) was used to extract total RNA from cell cultures, with GES-1 as the control, then cDNA was obtained by RT using PrimeScript cDNA qPCR RT kit (Thermo Fisher Scientific, Inc.), according to the manufacturer's protocol. Then, the acquired cDNA was used as a template to amplify double-stranded DNA using FastStart Universal SYBR-Green Master mix (Shanghai GenePharma, Co., Ltd.). Primers used for qPCR were as follows: $\beta$-actin (upstream, 5'-CTACAATGA GCTGCGTGTGG-3' and downstream, 5'-TAGCTCTTCTCC AGGGAGGA-3', 221 bp), RASAL1 (upstream, 5'-TGGATT TCTCTTCTTGCGATTCT-3' and downstream, 5'-TGTTGG TCCCGAAGGTCAA-3', 72 bp), miR-130b (upstream, 5'-GCC GCCAGTGCAATGATGAA-3' and downstream, 5-GTGCAG GGTCCGAGGT-3'); and U6 (upstream, 5'-CGCTTCGGCAGC ACATATACTA-3' and downstream, 5'-CGCTTCACGAAT TTGCGTGTCA-3'). The reaction conditions were as follows: $95^{\circ} \mathrm{C}$ for $2 \mathrm{~min}, 40$ cycles at $95^{\circ} \mathrm{C}$ for $30 \mathrm{sec}, 58^{\circ} \mathrm{C}$ for $30 \mathrm{sec}$ and $72^{\circ} \mathrm{C}$ for $1 \mathrm{~min}$, using an ABI Step One PCR system (Applied Biosystems; Thermo Fisher Scientific, Inc.). The expression of U6 was used as internal control. The relative expression of miR-130b-5p and RASAL1 compared with U6 was calculated using the $2^{-\Delta \Delta C q}$ method (10). The experiment was repeated three times.
Western blotting detection of RASAL1. To demonstrate the effect of miR-130b-5p on RASAL1 protein expression, MGC803 cells were transfected with miR-130b-5p mimics or miR-130b-5p inhibitor, with cells transfected with negative control (NC) sequence as the control. The transfected cells were collected, dissociated and incubated with radioimmunoprecipitation assay buffer (Beyotime Institute of Biotechnology, Shanghai, China) for protein extraction. Protein concentration was measured by the bicinchoninic acid (BCA) method. The expression of RASAL1 protein was measured by western blot analysis. Protein supernatants were separated via 10\% SDS-PAGE (Invitrogen; Thermo Fisher Scientific, Inc.) and transferred to nitrocellulose membranes (placed in ice water with a constant current of $100 \mathrm{~mA}$ overnight). The antibody was blocked with $5 \%$ skim milk at $4^{\circ} \mathrm{C}$ overnight. Rabbit anti-human RASAL1 polyclonal antibody (ab214321, 1:1,000; Abcam, Cambridge, UK) was used as the primary antibody and goat anti-rabbit- $\operatorname{IgG}(\mathrm{H}+\mathrm{L})$ antibody conjugated with horseradish peroxidase (ab205718, 1:5,000; Abcam) was used as the secondary antibody (and incubated for one hour at room temperature). $\beta$-actin (ab8226; Abcam) served as the internal control. The enhanced chemiluminescence imaging method was used to facilitate the detection of protein bands. The experiment was repeated three times.

Luciferase reporter gene assay. The 293T cells (Ribobio Co., Ltd., Guangzhou, China) were cultured in an incubator at $37^{\circ} \mathrm{C}$ and $5 \% \mathrm{CO}_{2}$. The pGL3-promoter vector containing the RASAL1 3'UTR and the control plasmid psiC-2 was constructed by Guangzhou Ribobio Co., Ltd., and then transfected with miR-130b-5p-mimics and NC, with NC as control. Then, logarithmic phase cells were plated onto 96 -well plates at a density of $1.5 \times 10^{4}$ cells/well and incubated at $37^{\circ} \mathrm{C}$ for $24 \mathrm{~h}$. Then, $293 \mathrm{~T}$ cells were transfected with pGL3 promoter RASAL1 and miR-130b-5p-mimics and NC seperately, using Lipofectamine 2000 (Invitrogen; Thermo Fisher Scientific, Inc.). At 6 h, complete medium was injected into the cell culture media and incubated at $37^{\circ} \mathrm{C}$ for $48 \mathrm{~h}$ to achieve high transfection efficiency. Then, cells were collected by the trypsin digestion method. A mixture of $100 \mu \mathrm{l}$ cell lysate and $100 \mu \mathrm{l}$ luciferase detection reagent (Beyotime Institute of Biotechnology) per sample was used to measure luciferase activity in relative light units, using a GLOMAX 20/20 luminometer (Promega Corporation, Madison, WI, USA), according to the manufacturer's protocol. The cell lysis solution was used as a blank control. The fluorescence value of hRluc (Hren-luciferase gene) was compared with that of hLuc (Firefly luciferase gene); the fluorescence ratio of the experimental group and the control group were analyzed. Each experiment was repeated at least three times.

Colony formation assay. Cells transfected with miR-130b-5p mimics, inhibitor and NC (control group) were used to evaluate the effect of abnormal miR-130b-5p expression on proliferation ability in gastric cancer cells. Cells from each group were seeded into 6 -well plates (1,000 cells/well) and incubated for 1 week in RPMI-1640 medium with $10 \% \mathrm{FBS}, 100 \mu \mathrm{g} / \mathrm{ml}$ streptomycin, and $100 \mathrm{U} / \mathrm{ml}$ penicillin. Cells from each group were stained with crystal violet for $30 \mathrm{~min}$ at room temperature. Clones $>2 \mathrm{~mm}$ were counted, and the result was calculated as the mean of three wells. Each experiment was repeated at least three times. 

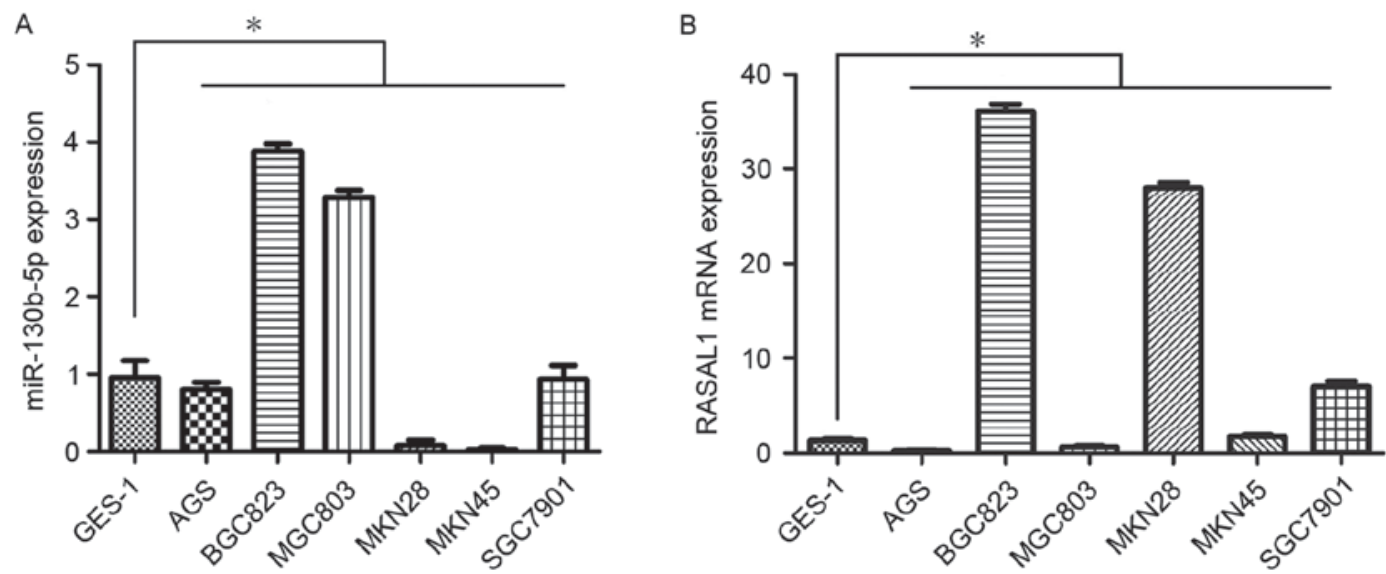

Figure 1. miR-130b-5p expression and RASAL1 mRNA expression in seven gastric cell lines. Expression of miR-130b-5p and RASAL1 at the mRNA level was evaluated by reverse transcription-quantitative polymerase chain reaction. GES-1 cells were used as the control. (A) miR-130b-5p expression in MGC803 cells and BGC823 cells was higher compared with the rest of the gastric cell lines studied. (B) RASAL1 mRNA expression was lower in MGC803 cells compared with other cell lines. ${ }^{*} \mathrm{P}<0.05$. miR, microRNA; RASAL1, RAS protein activator like 1.

Cell proliferation assay. Cells transfected with miR-130b-5p mimics, miR-130b-5p inhibitor and $\mathrm{NC}$ (control group) were seeded in a 96--well plate at a density of $2 \times 10^{3}$ cells/well, and incubated for $24 \mathrm{~h}$ at $37^{\circ} \mathrm{C}$ for adherence. MTT solution (20 $\mu \mathrm{l}$ in $200 \mu \mathrm{l}$ media) was added and incubated for $4 \mathrm{~h}$ at $37^{\circ} \mathrm{C}$. This was followed by removal of the remaining liquid and injection of $150 \mu \mathrm{l}$ DMSO per well. The formazan crystals were dissolved by gentle shaking of the plate for $5 \mathrm{~min}$ at room temperature. The absorbance was measured at $540 \mathrm{~nm}$ using a multi-scan plate reader. Each experiment was repeated at least three times.

Transwell assays. MGC803 cells transfected with miR-130b-5p mimics and inhibitor were incubated at $37^{\circ} \mathrm{C}$ and $5 \% \mathrm{CO}_{2}$, with cells transfected with $\mathrm{NC}$ as the control. Matrigel (BD Biosciences, Franklin Lakes, NJ, USA) was combined with serum-free RPMI-1640 medium (Gibco; Thermo Fisher Scientific, Inc.). For the invasion assay, the Transwell chamber contained Matrigel. For the migration assay, no Matrigel was used. Cells from each group were trypsinized, centrifuged $(1,000 \mathrm{rpm}, 167.7 \mathrm{x} \mathrm{g}$ for $5 \mathrm{~min}$ at room temperature) and resuspended in serum-free medium at a concentration of $5 \times 10^{5} / \mathrm{ml}$. Serum-free medium $(50 \mu \mathrm{l})$ was added to the upper well of the Transwell chamber containing $2.5 \times 10^{3}$ cells/per well (Corning, Inc., Corning, NY, USA) with RPMI-1640 medium (containing $10 \%$ serum) in the lower site. Following incubation for $48 \mathrm{~h}$, cells in the upper chamber were removed and cells in the lower chamber were stained with crystal violet at room temperature for $30 \mathrm{~min}$. Finally, cells in the lower chamber were counted under a light microscope. Each experiment was repeated at least three times.

Statistical analysis. All experimental data were analyzed using SPSS 18.0 software (SPSS, Inc., Chicago, IL, USA) and presented as the mean \pm standard deviation. Comparisons between experimental and control groups were performed using Student's t-tests. Significant differences among groups were assessed by one-way analysis of variance followed by Tukey's post hoc test for multiple comparisons. $\mathrm{P}<0.05$ was considered to indicate a statistically significant difference.

\section{Results}

Expression of miR-130b-5p and RASAL1 in seven gastric cell lines. Expression of miR-130b-5p and RASAL1 was assessed by RT-qPCR in seven gastric cell lines. The results demonstrated higher expression of miR-130b-5p in BGC823 cells and MGC803 cells compared with the rest of the gastric cell lines studied (Fig. 1A). Furthermore, consistent with the higher expression of miR-130b-5p, the expression of RASAL1 was markedly decreased in MGC803 cells compared with other cell lines (Fig. 1B). As a result, the MGC803 cell line was selected for subsequent experiments.

RASAL1 is a target gene of miR-130b-5p. In order to reveal the molecular mechanism of miR-130b-5p in gastric cancer, bioinformatics software (FindTar3, version 3.11.12; https://bio.sz.tsinghua.edu.cn) was used to predict the targeted interaction between RASAL1 and miR-130b-5p. The 8-29 and 684-707 loci of the 3'-UTR of RASAL1 were predicted to be a complementary target of miR-130b-5p. Based on this prediction, a luciferase reporter gene assay was used to evaluate the interaction between RASAL1 and miR-130b-5p. A significant decrease in luciferase activity was observed in cells co-transfected with miR-130b-5p and pGL3-RASAL1 compared with the other three groups $(\mathrm{P}<0.05$; Fig. 2A). These results demonstrated that RASAL1 may be regulated by miR-130b-5p. To verify these findings, the mRNA and protein expression of RASAL1 was measured in cells transfected with miR-130b-5p mimics, miR-130b-5p inhibitor and NC by RT-qPCR and western blot analysis. The results indicated decreased expression of RASAL1 at the mRNA protein levels in cells transfected with miR-130b-5p mimics $(\mathrm{P}<0.05)$ and a significant increase in RASAL1 in cells transfected with miR-130b-5p inhibitor $(\mathrm{P}<0.05)$, compared with cells transfected with NC (Fig. 2B and C).

Cell proliferation assay. MTT assay and low-density colony formation experiments were used to evaluate the effect of miR-130b-5p on MGC803 cell proliferation. The results revealed a significantly higher clone formation ability in 
A

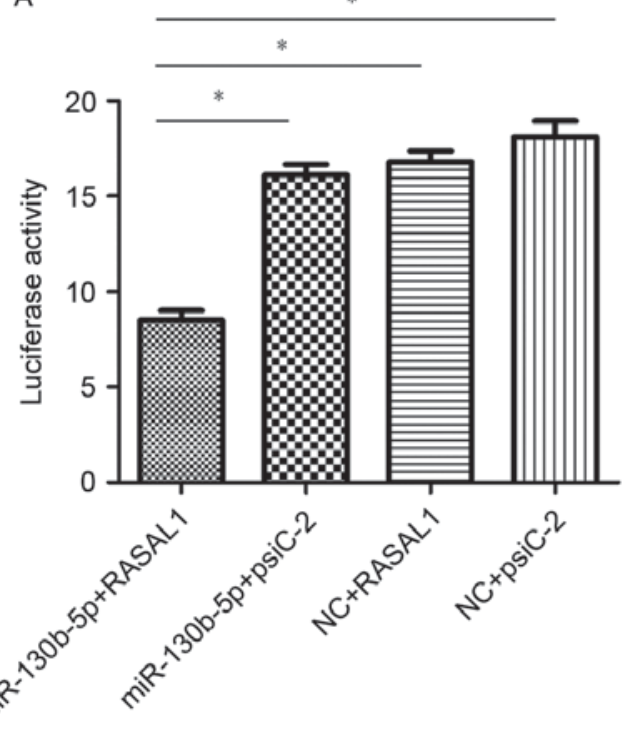

B

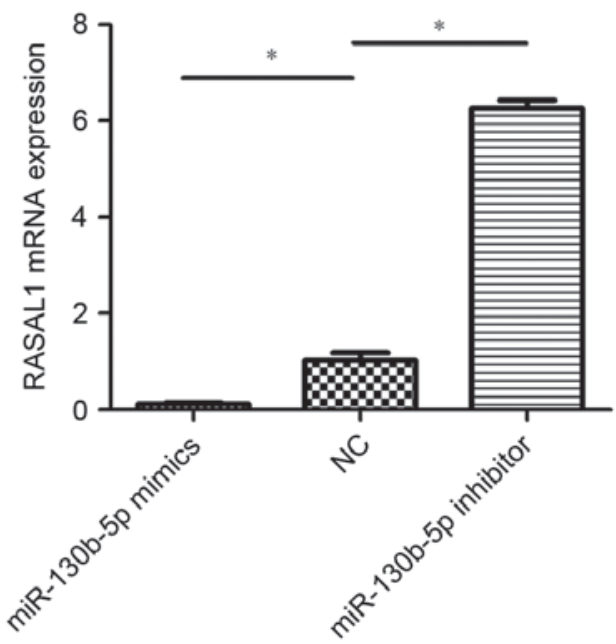

C

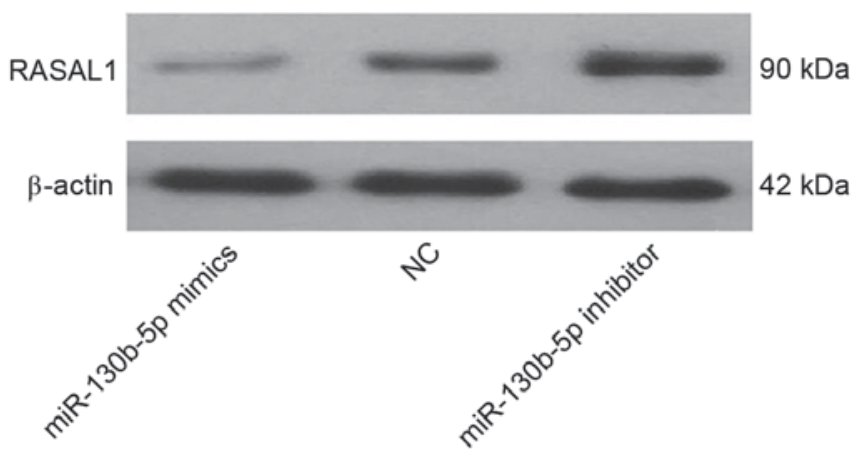

Figure 2. Effect of miR-130b-5p on RASAL1. (A) Luciferase reporter gene assay indicated lower RLU values in cells co-transfected with miR-130b-5p mimics (with concentration of $50 \mathrm{nM}$ ) and pGL3-RASAL1 (with concentration of $50 \mathrm{nM}$ ) in comparison with cells in other three groups (cells co-transfected with miR-130b-5p and psiC-2, cells co-transfected with NC and pGL3-RASAL1, and cells co-transfected with NC and psiC-2) (psiC-2 was used as control plasmid with concentration of $50 \mathrm{nM}$ ). (B) Reverse transcription-quantitative polymerase chain reaction analysis of mRNA expression of RASAL1 in cells transfected with miR-130b-5p mimics and miR-130b-5p inhibitor. (C) Western blot analysis of protein expression of RASAL1 in cells transfected with miR-130b-5p mimics and miR-130b-5p inhibitor. "P<0.05. miR, microRNA; RASAL1, RAS protein activator like 1; NC, negative control.

cells transfected with miR-130b-5p mimics (P<0.05; Fig. 3) and a significantly lower clone formation ability in cells transfected with miR-130b-5p inhibitor $(\mathrm{P}<0.05$; Fig. 4) compared with the $\mathrm{NC}$ group. Consistent with this, OD values indicated a significantly higher rate of proliferation in cells transfected with miR-130b-5p mimics $(\mathrm{P}<0.05$; Fig. 5) and a significantly lower rate of proliferation in cells transfected with miR-130b-5p inhibitor ( $\mathrm{P}<0.05$; Fig. 6) compared with the NC group at 48, 72 and $96 \mathrm{~h}$. These results indicated an increased capacity for cell proliferation following overexpression of miR-130b-5p and a weakened capacity following downregulation of miR-130b-5p.

Measurement of migration and invasion. Transwell assays were performed in order to evaluate the effect of miR-130b-5p on the migration and invasion abilities of MGC803 cells. The data demonstrated a significantly increased migration and invasion ability in cells transfected with miR-130b-5p mimics $(\mathrm{P}<0.05$; Fig. 7$)$, and a significantly decreased migration and invasion ability in cells transfected with miR-130b-5p inhibitor ( $\mathrm{P}<0.05$; Fig. 8) compared with cells transfected with NC.

\section{Discussion}

The association between RAS gene and occurrence of cancer was gradually taken seriously in recent studies. The RAS-RAF-MEK-ERK signal transduction pathway performs a key function in cell proliferation, migration and invasion (11). In the process of regulation of cell proliferation, migration and invasion, the product of the activated RAS gene (RAS protein) is a key controlling element in the RAS-RAF-MEK-ERK signal transduction pathway. It has been reported that RAS gene mutations eventually lead to abnormal RAS hyperactivity, excessive cell growth and accelerated development of tumors (12).

RAS proteins (Ras, Rho, Rab, Arf, Sarl and Ran families) are an element of GTP hydrolases (G proteins) that regulate and control the RAS hyperactivity. The RAS protein exists in two forms; an active form bound with GTP and an inactive form bound with GDP. The transformation of RAS protein structures is closely associated with the capacity for cell proliferation, migration and invasion in gastric cell lines (5). There are two types of molecule involved in the conversion between the active and inactive forms of RAS protein: RAS GAPs and RAS GEFs. The function of RAS GEFs is 


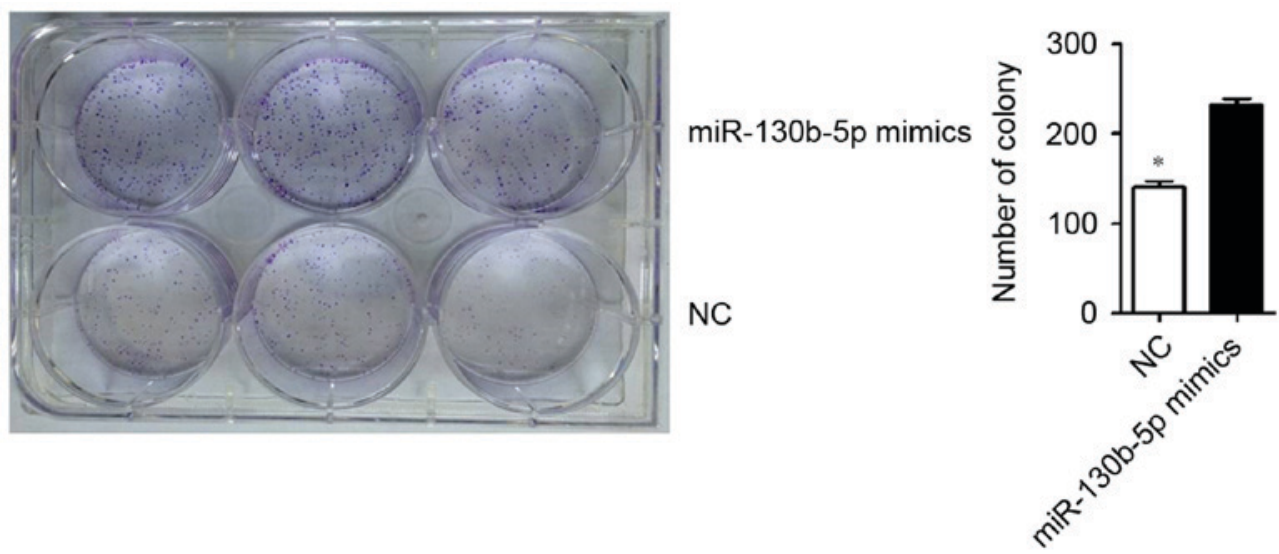

Figure 3. Upregulation of miR-130b-5p stimulates clone formation ability in MGC803 cells. Cell proliferation was evaluated by a low-density clone formation experiment following transfection with miR-130b-5p mimics and NC. "P $<0.05$. miR, microRNA; NC, negative control.

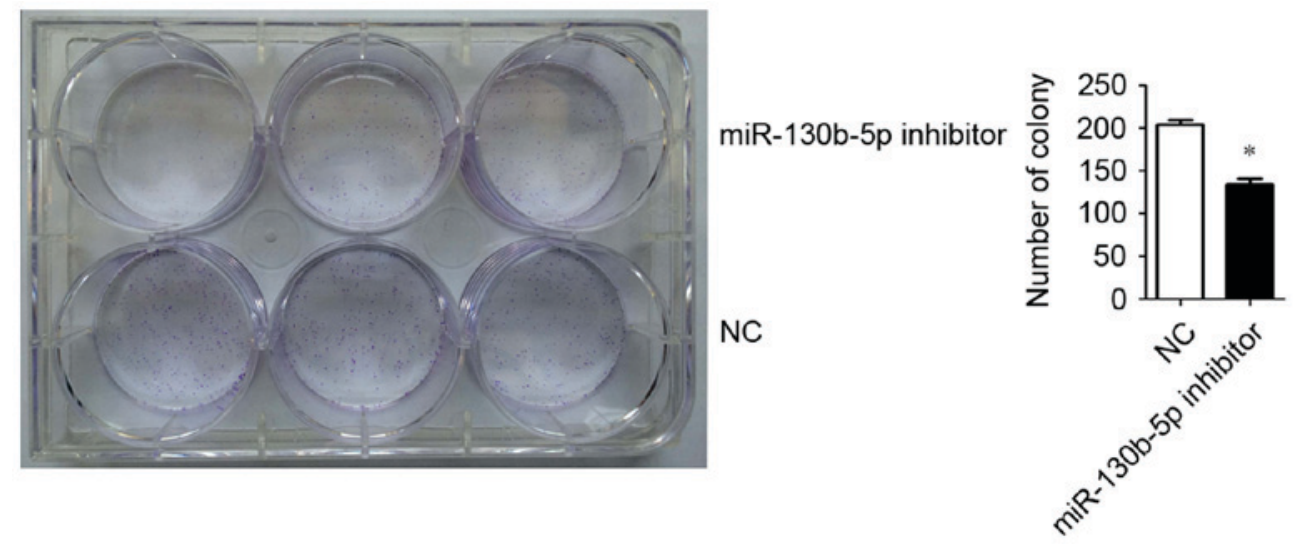

Figure 4. Downregulation of miR-130b-5p decreases clone formation ability in MGC803 cells. Cell proliferation was evaluated by a low-density clone formation experiment following transfection with miR-130b-5p inhibitor and NC. " $\mathrm{P}<0.05$. miR, microRNA; NC, negative control.

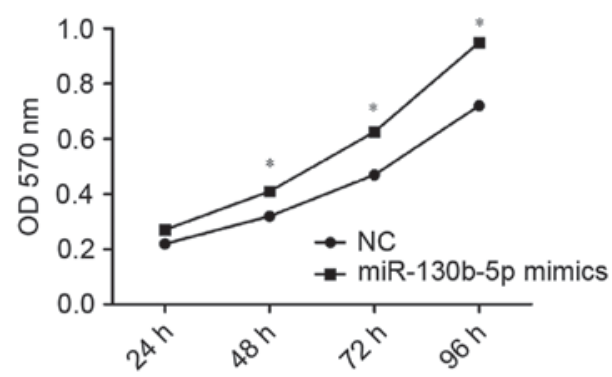

Figure 5. Upregulation of miR-130b-5p stimulates proliferation capacity of MGC803 cells. Cell proliferation was measured by MTT assay following transfection with miR-130b-5p mimics and NC. ${ }^{*} \mathrm{P}<0.05$ vs. NC. miR, microRNA; NC, negative control; OD, optical density.

to facilitate binding with GTP and eventually lead to the activation of RAS, whereas RAS GAPs promote the conversion from GTP binding to GDP binding and ultimately give rise to inactivation of RAS (13). RASAL1 is a key member of the RAS GAP family (14). RASAL1 is thought to act as a tumor suppressor gene by promoting the conversion from GTP binding (active) to GDP binding (inactive), eventually suppressing tumorigenesis in multiple types of cancer (15). A previous study indicated that various types of cancer are associated with low expression of the RASAL1 gene, including

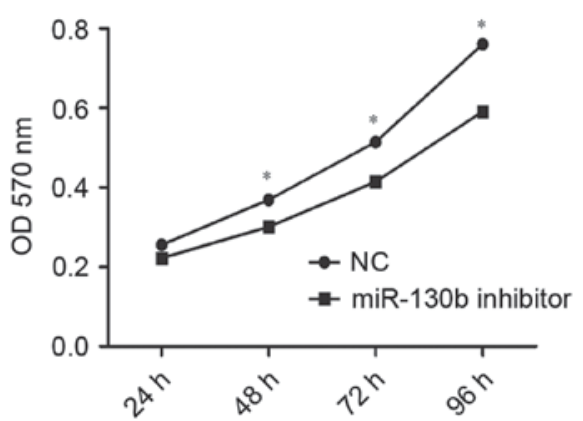

Figure 6. Downregulation of miR-130b-5p decreases proliferation capacity of MGC803 cells. Cell proliferation was measured by MTT assay following transfection of miR-130b-5p inhibitor and NC. ${ }^{*} \mathrm{P}<0.05$ vs. miR-130b inhibitor. miR, microRNA; NC, negative control; OD, optical density.

nasopharyngeal carcinoma, breast cancer, lung cancer, liver cancer and esophageal cancer (13).

Our previous research indicated that overexpression of RASAL1 may attenuate gastric carcinogenesis in vivo and in vitro (9). In other words, RASAL1 is a tumor suppressor gene and low expression of RASAL1 may result in RAS hyperactivity, leading to the occurrence of malignant tumors. miR-130b-5p is a small non-coding RNA situated in the $22 \mathrm{q} 11$ locus. Numerous studies have indicated that miR-130b 

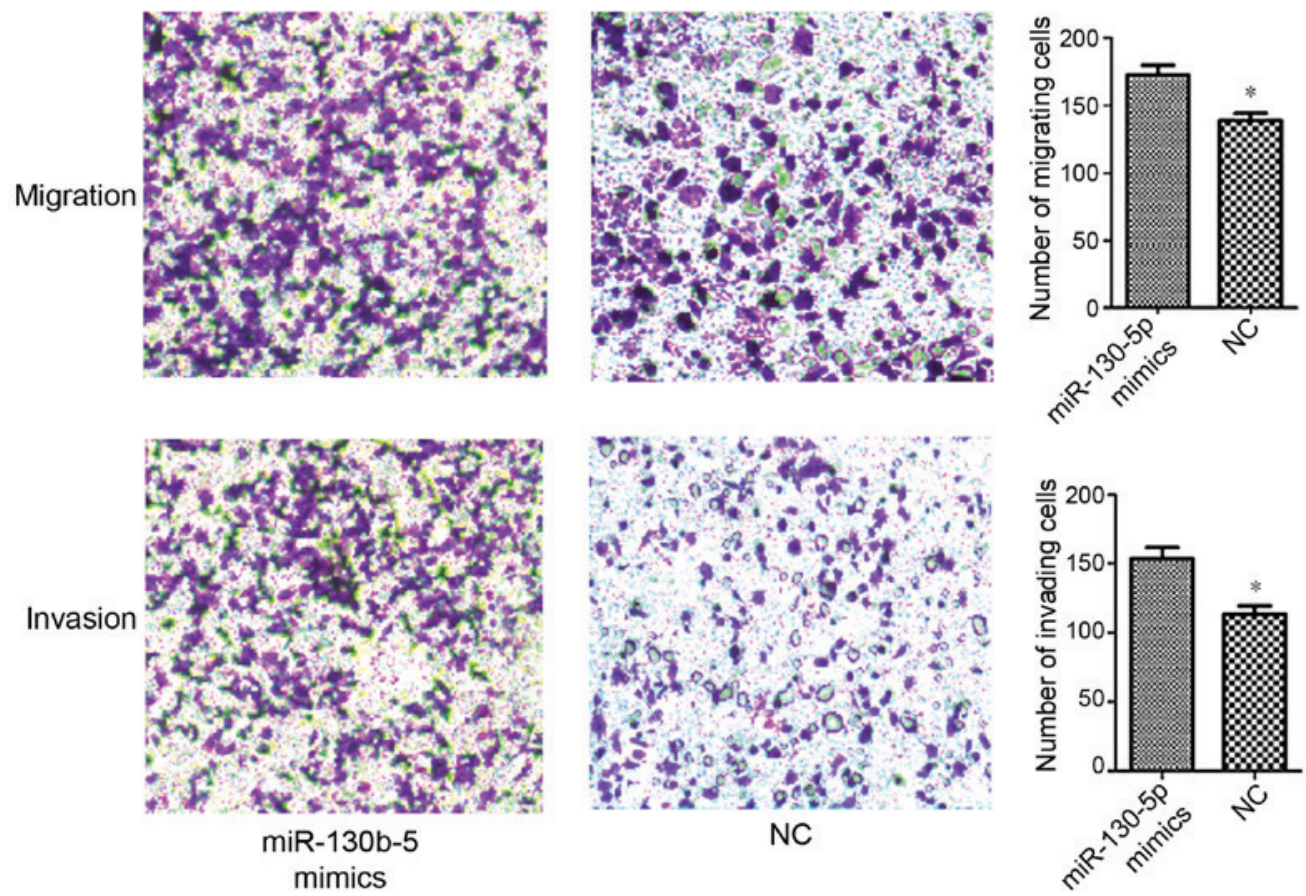

Figure 7. Upregulation of miR-130b-5p stimulates the migration and invasion ability of MGC803 cells (magnification, x100). Transwell and Matrigel assays were performed to measure migration and invasion abilities of MGC803 cells. " $\mathrm{P}<0.05$ vs. miR-130-5p mimics. miR, microRNA; NC, negative control.
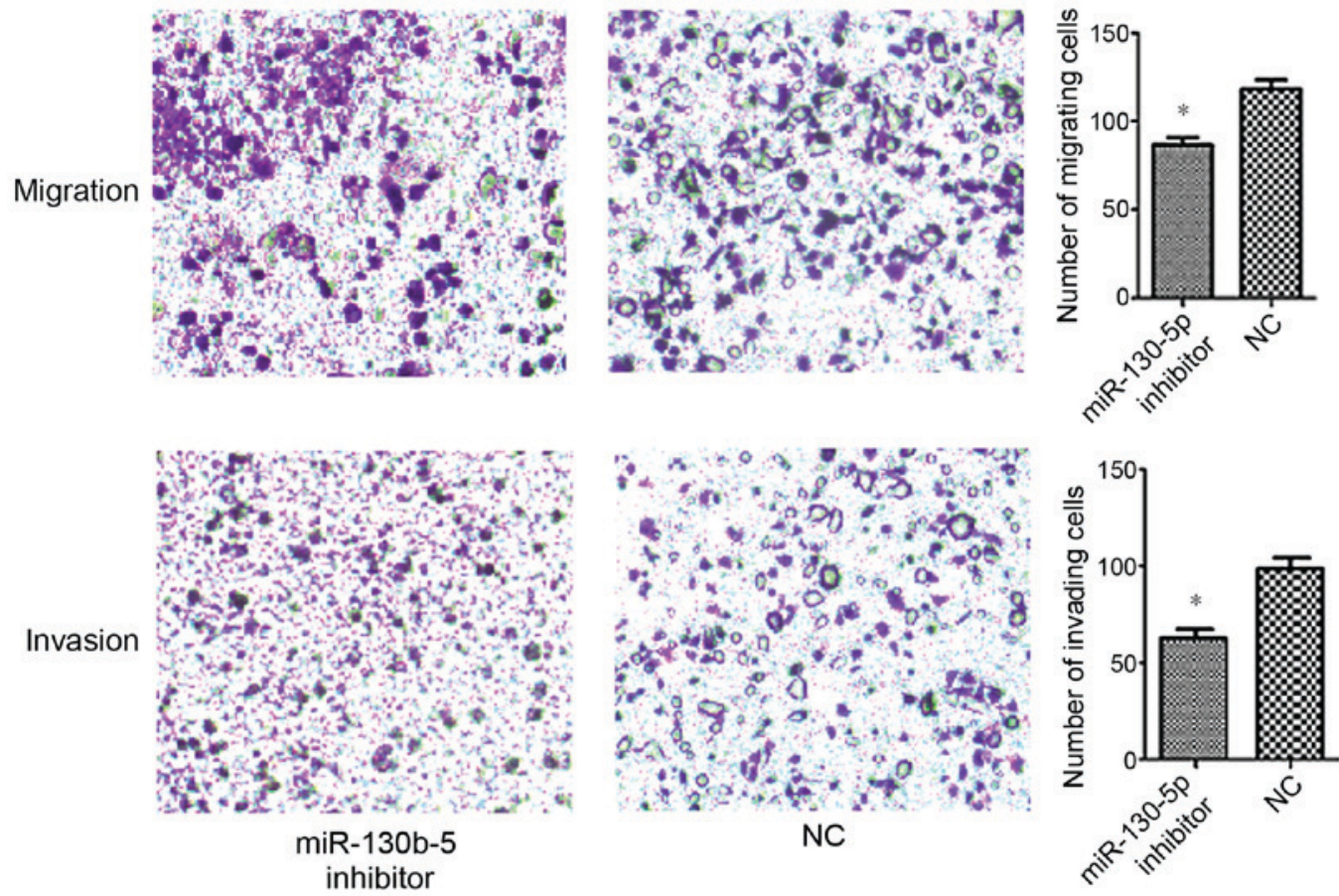

Figure 8. Downregulation of miR-130b-5p decreases the migration and invasion ability of MGC803 cells (magnification, x100). Transwell and Matrigel assays were performed to measure migration and invasion abilities of MGC803 cells. ${ }^{*} \mathrm{P}<0.05$ vs. NC. miR, microRNA; NC, negative control.

is overexpressed in various types of tumor, including gastric cancer, liver cancer and endometrial cancer (16-18). It was verified that the oncogenic role of miR-130b-5p is achieved by means of downregulation of a diverse set of cancer suppressor genes, including phosphatase and tensin homolog serve protective roles against esophageal cancer (19). In the present study, bioinformatics software (FindTar3) was used to predict RASAL1 as an miR-130b-5p target gene and a luciferase reporter gene assay was used to verify the targeted interaction between miR-130b-5p and RASAL1. In addition, a low level of RASAL1 expression was observed by RT-qPCR and western blot analysis following transfection with miR-130b-5p mimics. Conversely, a high level of RASAL1 expression was observed in cells transfected with miR-130b-5p inhibitor. The results also demonstrated that the level of miR-130b-5p was higher in MGC803 cells compared with other gastric cell lines. A high 
level of miR-130b-5p expression accelerated RAS hyperactivity and suppressed the expression of RASAL1 gene, eventually resulting in tumorigenesis. On the other hand, downregulated miR-130b-5p in cells transfected with miR-130b-5p inhibitor was demonstrated to exhibit a protective effect by accelerating the expression of cancer suppressor gene RASAL1. It has been reported that activation of the RAS-RAF-MEK-ERK transduction pathway is of vital importance in cell proliferation, migration and invasion (9). The current data demonstrated that a high level of miR-130b-5p expression could restrain the expression of RASAL1 and accelerate the proliferation, invasion and migration ability of MGC803 cells. By contrast, increased RASAL1 and reduced ability of proliferation, invasion and migration were observed in cells with downregulated miR-130b-5p.

In conclusion, the present study described the miR-130b-5p/ RASAL1 axis and provided a potential mechanism for RAS hyperactivity. In addition, the present study identified the effect of miR-130b-5p in gastric cell proliferation, migration and invasion. It has been demonstrated that miR-130b-5p is involved in the occurrence and development of gastric cancer by targeting RASAL1. These results offer a potential basis for novel targeted therapy in gastric cancer. The current findings will be explored further via experimentation on animals in future studies.

\section{Acknowledgements}

Not applicable.

\section{Funding}

The present study was supported by the Research Project of National Health and Family Planning Commission of China (grant no. W201305) and the Natural Science Foundation of Jiangsu Province of China (grant no. BK2008301).

\section{Availability of data and materials}

The datasets used and/or analyzed during the current study are available from the corresponding author on reasonable request.

\section{Authors' contributions}

HC designed the study. YY performed the experiments. DS and JZ conducted luciferase assays. QY conducted the cell formation assay. YY and JW analyzed the data.

\section{Ethics approval and consent to participate}

Not applicable.

\section{Consent for publication}

Not applicable.

\section{Competing interests}

The authors declare that they have no competing interests.

\section{Authors' information}

Professor Hong Chen, Department of Gastroenterology, Zhongda Hospital Medical School, Southeast University, 87 Dingjiaqiao, Gulou, Nanjing, Jiangsu 210000, P.R. China

\section{References}

1. Shin JY, Kim YI, Cho SJ, Lee MK, Kook MC, Lee JH, Lee SS, Ashktorab H, Smoot DT, Ryu KW, et al: MicroRNA 135a suppresses lymph node metastasis through down-regulation of ROCK1 in early gastric cancer. PLoS One 9: e85205, 2014

2. Song Z, Wu Y, Yang J, Yang D and Fang X: Progress in the treatment of advanced gastric cancer. Tumour Biol 39, 2017.

3. Wittekind C: The development of the TNM classification of gastric cancer. Pathol Int 65: 399-403, 2015.

4. Kim SJ, Wang YG, Lee HW, Kang HG, La SH, Choi IJ, Irimura T, Ro JY, Bresalier RS and Chun KH: Up-regulation of neogenin-1 increases cell proliferation and motility in gastric cancer. Oncotarget 5: 3386-3398, 2014.

5. Chen H, Cheng ZY, Pan Y, Wang Z, Liu Y and Zhang JQ: RASAL1 influences the proliferation and invasion of gastric cancer cells by regulating the RAS/ERK signaling pathway. Hum Cell 27: 103-110, 2014.

6. Gong B, Liu WW, Nie WJ, Li DF, Xie ZJ, Liu C, Liu YH, Mei P and Li ZJ: MiR-21/RASA1 axis affects malignancy of colon cancer cells via RAS pathways. World J Gastroenterol 21: 1488-1197, 2015.

7. Hao NB, He YF, Li XQ, Wang K and Wang RL: The role of miRNA and lncRNA in gastric cancer. Oncotarget 8: 81572-81582, 2017.

8. Tu K, Zheng X, Dou C, Li C, Yang W, Yao Y and Liu Q: MicroRNA-130b promotes cell aggressiveness by inhibiting peroxisome proliferator-activated receptor gamma in human hepatocellular carcinoma. Int J Mol Sci 15: 20486-20499, 2014.

9. Chen H, Zhao JY, Qian XC, Cheng ZY, Liu Y and Wang Z: RASAL1 attenuates gastric carcinogenesis in nude mice by blocking RAS/ERK signaling. Asian Pac J Cancer Prev 16: 1077-1082, 2015.

10. Livak KJ and Schmittgen TD: Analysis of relative gene expression data using real-time quantitative PCR and the 2(-Delta Delta C(T)) method. Methods 25: 402-408, 2001.

11. McCubrey JA, Steelman LS, Chappell WH, Abrams SL, Wong EW, Chang F, Lehmann B, Terrian DM, Milella M, Tafuri A, et al: Roles of the Raf/MEK/ERK pathway in cell growth, malignant transformation and drug resistance. Biochim Biophys Acta 1773: 1263-1284, 2007.

12. Chen H, Pan Y, Cheng ZY, Wang Z, Liu Y, Zhao ZJ and Fan H: Hypermethylation and Clinicopathological significance of RASAL1 gene in gastric cancer. Asian Pac J Cancer Prev 14: 6261-6265, 2013.

13. Jin $\mathrm{H}$, Wang $\mathrm{X}$, Ying J, Wong AH, Cui Y, Srivastava $\mathrm{G}$, Shen ZY, Li EM, Zhang Q, Jin J, et al: Epigenetic silencing of a $\mathrm{Ca}(2+)$-regulated Ras GTPase-activating protein RASAL defines a new mechanism of Ras activation in human cancers. Proc Natl Acad Sci USA 104: 12353-12358, 2007.

14. Calvisi DF, Ladu S, Conner EA, Seo D, Hsieh JT, Factor VM and Thorgeirsson SS: Inactivation of Ras GTPase-activating proteins promotes unrestrained activity of wild-type Ras in human liver cancer. J Hepatol 54: 311-319, 2011.

15. Bernards A and Settleman J: Loss of the Ras regulator RASAL1: Another route to Ras activation in colorectal cancer. Gastroenterology 136: 46-48, 2009.

16. Lai KW, Koh KX, Loh M, Tada K, Subramaniam MM, Lim XY, Vaithilingam A, Salto-Tellez M, Iacopetta B, Ito Y, et al: MicroRNA-130b regulates the tumour suppressor RUNX3 in gastric cancer. Eur J Cancer 46: 1456-1463, 2010.

17. Li BL, Lu C, Lu W, Yang TT, Qu J, Hong X and Wan XP: miR-130b is an EMT-related microRNA that targets DICER1 for aggression in endometrial cancer. Med Oncol 30: 484, 2013.

18. Colangelo T, Fucci A, Votino C, Sabatino L, Pancione M, Laudanna C, Binaschi M, Bigioni M, Maggi CA, Parente D, et al: MicroRNA-130b promotes tumor development and is associated with poor prognosis in colorectal cancer. Neoplasia 15: 1086-1099, 2013.

19. Yu T, Cao R, Li S, Fu M, Ren L, Chen W, Zhu H, Zhan Q and Shi R: MiR-130b plays an oncogenic role by repressing PTEN expression in esophageal squamous cell carcinoma cells. BMC Cancer 15: 29, 2015. 